\title{
Niveau de parasitisme des hévéas par les Loranthaceae dans la région du Sud-ouest Cameroun
}

\author{
NGOTTA BIYON Jacques Bruno ${ }^{(1)^{*}}$ DIBONG Siegfried Didier ${ }^{(1,2,3)}$, TAFFOUO Victor Désiré $(1,2)$, ONDOUA \\ Joseph Marie (4), Bilong Paul(5) \\ ${ }^{1}$ Département de Biologie des Organismes Végétaux, Faculté des Sciences, B.P. 24157, Université de Douala, \\ Cameroun. \\ 2 Institut des Sciences Halieutiques, B.P. 2701, Université de Douala, Cameroun. \\ 3. Département des Sciences Pharmaceutiques, Faculté de Médecine et des Sciences Pharmaceutiques, B.P. 2701, \\ Université de Douala, Cameroun. \\ 4. Département de Botanique et de Physiologie Végétale, Faculté des Sciences, B. P. 63, Université de Buea, Cameroun. \\ ${ }^{5}$ Département des Sciences de la Terre, Faculté des Sciences, B. P. 47, Université de Yaoundé I, Cameroun \\ * Auteur de la correspondance : ngottabruno@gmail.com
}

Original submitted in on $4^{\text {th }}$ November 2015. Published online at www.m.elewa.org on 31st December 2015 http://dx.doi.org/10.4314/jab.v96i1.3

\section{RESUME}

Objectif : La présente étude s'intéresse au parasitisme des hévéas par les Loranthaceae dans les plantations de la Cameroon Development Coorporation (CDC) de la région du Sud-ouest du Cameroun.

Méthodologie et résultats : L'étude a consisté à dénombrer les espèces de Loranthaceae parasitant les hévéas, le nombre de touffes par individu parasité afin de déterminer le taux ainsi que l'intensité du parasitisme dans les plantations. Les résultats montrent qu'une seule espèce de Loranthaceae a été rencontrée dans les parcelles étudiées, Phragmanthera capitata. Le taux de parasitisme varie significativement du village Sonne $(65 \%)$ au village Likomba $(51,53 \%)$. Le clone PB 217 est le plus -parasité $(65,08 \%)$, tandis que le clone PR 107 est le moins parasité (48,31\%).

Conclusion et application des résultats: Les types de voies d'accès jouent un rôle important dans la propagation du parasitisme. II serait judicieux dans un premier temps, afin de mieux contrôler le parasitisme, de planter les clones les moins sensibles en bordure des plantations. Toutefois, des méthodes de lutte efficaces devront être mises sur pied afin d'améliorer les rendements des plantations.

Mots-clés : Hévéas, Loranthaceae, parasitisme, sud-ouest.

\section{Parasitism of rubber trees by Loranthaceae in the South-west region of Cameroon ABSTRACT}

Objective: The present work consists in looking for characterizing the parasitism of the rubber trees by the Loranthaceae in the plantations of the Cameroon Development Corporation (CDC) of the Southwest of Cameroon region.

Methodology and results: The study consisted in counting the various species of Loranthaceae which lives as parasite on the seed fields, on the feet of infested rubber trees, and to determine the intensity of the parasitism of rubber trees. Results showed that only one species of Loranthaceae has been met in the studied areas, 
Phragmanthera capitata. The rate of parasitism varies from Sonne (65\%) to Likomba villages $(51,53 \%)$. The clone PB 217 was the most parasitized (65,08\%), while the clone PR 107 was the less parasitized $(48,31 \%)$. Conclusion and application of the results: The types of approach paths play an important role in the propagation of the parasitism. The clones least sensitive to the parasitism should be plant in border of the plantation to reduce the level of parasitism. However, appropriate control methods against the parasitism by Loranthaceae should be sought to increase plantation yields.

Key-words : Loranthaceae, parasitism, rubber tree, south-west.

\section{INTRODUCTION}

Les Loranthaceae sont de petits arbrisseaux épiphytes chlorophylliens, qui vivent en hémiparasites sur les branches d'arbres et d'arbustes. Elles appartiennent au groupe des Angiospermes parasites, ne représentant que $2 \%$ des plantes à graines (Raynal-Roques \& Paré, 1998). Ces plantes se présentent sous forme de touffes. Elles sont liées à leur hôte par un véritable pont structural et physiologique qui constitue un système d'absorption ou suçoir, permettant au parasite de prélever l'eau, les substances minérales et le complément de matière organique dont il a besoin (Bannister et al., 2002). En plus des arbres sauvages, les Loranthaceae attaquent les essences cultivées, telles que cacaoyers, avocatiers, agrumes et hévéas, réduisant leur rendement (Sonké et al., 2000). Dans les conditions écologiques extrêmes du

\section{MATERIEL ET METHODES}

Présentation de la zone d'étude : La zone d'étude est située dans les plantations de la Cameroon Development Coorporation (CDC) du sud-ouest, aux alentours des localités Tiko et Muyuka. La CDC est une société agro industrielle localisée dans les régions du Littoral, du NordOuest et Sud-Ouest au Cameroun, et regroupe les plantations de bananeraies, de palmiers à huile et d'hévéas. Les plantations d'hévéas couvrent une superficie d'environ 21000 ha, organisés en 10 villages appelés "Estate" constitués de plusieurs blocs appelés "Section". Chaque bloc, de dimension variable renferme des arbres appartenant à un même clone et plantés la même année. Les arbres sont répartis en plusieurs lignes, espacées entre elles de 6 à $7 \mathrm{~m}$ (espace interligne) et les arbres situés sur une même ligne sont espacés de 2,7 à $3 \mathrm{~m}$ (espace intraligne).

Choix des parcelles et des clones : Les observations ont été effectuées dans la période allant de septembre 2014 à mai 2015 dans les blocs d'hévéa âgés de 25 ans. Ces blocs ont été choisis pour leur position en bordure de
Sahel, elles parasitent fortement les peuplements de karité, pouvant même entraîner leur mort (Boussim, 2002). La famille des Loranthaceae, largement répandue dans le monde, comprend 77 genres et 950 espèces. Sept genres et 26 espèces sont signalés au Cameroun (Polhill \& Wiens, 1998), notamment dans la région littorale (Dibong et al., 2009) où sont implantées de vastes plantations agroindustrielles. La présente étude dresse l'état du parasitisme par les Loranthaceae dans les plantations d'hévéas de la zone du sud-ouest. Les objectifs spécifiques ont consisté à dénombrer les parasites présents dans les plantations, déterminer le taux de parasitisme de ces plantations, et déterminer l'influence de la circonférence de l'arbre ainsi que celle des voies d'accès sur le parasitisme.

la route principale (la Nationale $\mathrm{N}^{\circ} 5$ ), des routes secondaires qui sont les routes d'accès aux plantations et aux routes tertiaires, qui sont les pistes existant à l'intérieur des plantations. Les clones étudiés à l'intérieur de ces plantations sont PB 217, PB 260 et PR 107. Ces clones ont été choisis pour leur forte présence et leur forte productivité dans la région du Sud-Ouest.

Méthodologie : Les observations ont été effectuées dans 4 villages, Likomba, Meanja, Misselele et Sonne. Les secteurs explorés sont Camp 6A, Meanja 2, Muyuka 1, Muyuka 2, Mondoni 2 et Sonne. Pour chaque arbre parasité, le nombre de parasite, le nombre de touffes du parasite, le numéro de ligne et la circonférence de l'arbre ont été notés. Ces éléments ont permis de déterminer le taux de parasitisme de la plantation, qui est le pourcentage d'arbres parasités obtenus; l'intensité de parasitisme qui est le nombre moyen de touffes du parasite observé par arbre ; la localisation du parasitisme qui est l'influence de la position de l'arbre sur le parasitisme ; l'effet de la circonférence de l'arbre sur le 


\section{Ngotta Biyon Bi et al. J. Appl. Biosci. $2015 \quad$ Niveau de parasitisme des hévéas par les Loranthaceae dans la région du Sud-ouest Cameroun}

parasitisme. Afin de mesurer l'impact des voies d'accès sur le parasitisme, des parcelles de 4 ha ont été choisies le long des routes principales, d'une largeur de $12,5 \mathrm{~m}$, le long des les routes secondaires, d'une largeur de 6,8 m, et les routes tertiaires d'une largeur de $3 \mathrm{~m}$.

Analyses statistiques : Les analyses statistiques ont été effectuées à l'aide du logiciel XLSTAT 2007. Le test de

\section{RESULTATS}

Loranthaceae rencontrées : Un total de 4625 arbres a été dénombré dans les parcelles étudiées. Une espèce de Loranthaceae a été rencontrée, Phragmanthera capitata. Cette Loranthaceae, aux fleurs en forme de

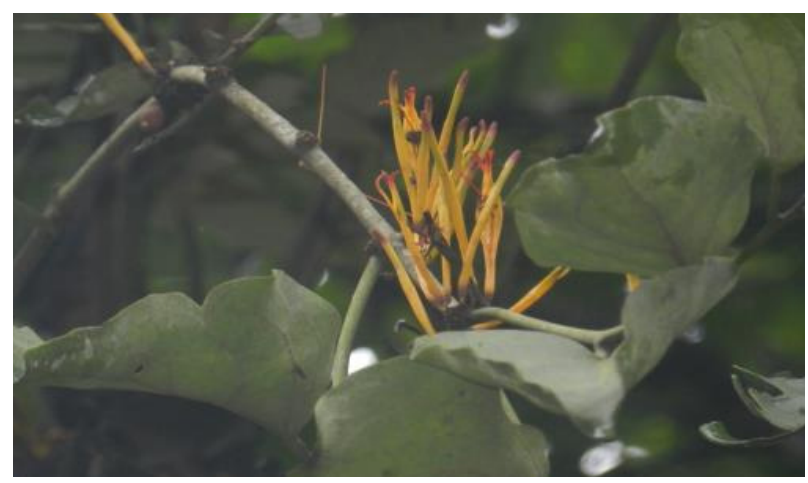

Figure 1 : Tige de Phragmanthera capitata portant des fleurs

Niveau de parasitisme des plantations: Le niveau global de parasitisme des plantations est de $57,85 \%$. Le taux de parasitisme varie significativement de $65 \%$ dans le village Sonne, à 51,53 \% à Likomba (Figure 2). Parmi les parcelles étudiées, la parcelle de Mondoni 2 possède le taux de parasitisme le plus élevé, avec un taux de 67,5 $\%$, tandis que la parcelle de Meanja 2 renferme le taux de parasitisme le moins élevé (Tableau 1). Le clone PB 217 possède un taux de parasitisme (65,8 \%)
Fischer a permis la comparaison des moyennes et des variances dans les parcelles étudiées. Les droites de régression ont permis de vérifier l'existence de relation entre la circonférence de l'arbre et le nombre de touffes du parasite.

buchettes d'allumettes, a été retrouvée dans toutes les parcelles étudiées (Figure 1). Cette espèce parasite également les plantes telles que l'avocatier, le cacaoyer ou encore les agrumes.

Tableau 1 : Inventaire des individus parasités dans les sites étudiés

\begin{tabular}{|l|l|l|r|r|r|r|r|}
\hline Bloc & Parcelle & Localisation & N.I.R & N.I.P & N.T.P & T.P & N.M.T \\
\hline LIKOMBA & CAMP 6A & Route secondaire & 561 & 307 & 746 & 54,72 & 2,43 \\
\hline & CAMP 6A & Route tertiaire & 1133 & 566 & 1274 & 49,96 & 2,25 \\
\hline MEANJA & MEANJA2 & Route secondaire & 592 & 286 & 1254 & 48,31 & 4,38 \\
\hline & MUYUKA1 & Route secondaire & 305 & 166 & 817 & 54,43 & 4,92 \\
\hline & MUYUKA2 & Route secondaire & 406 & 232 & 631 & 57,14 & 2,72 \\
\hline MISSELELE & MONDONI2 & Route secondaire & 332 & 224 & 857 & 67,47 & 3,83 \\
\hline & MONDONI2 & Route tertiaire & 416 & 260 & 619 & 62,5 & 2,38 \\
\hline SONNE & SONNE & Route principale & 880 & 572 & 1376 & 65 & 2,41 \\
\hline TOTAL & & & 4625 & 2613 & 7574 & & \\
\hline
\end{tabular}

N.I.R : Nombre d'individus recensés ; N.I.P : Nombre d'individus parasités ; N.T.P : Nombre de touffes du parasite ; T.P : Taux de parasitisme ; N.M.T : Nombre moyen de touffes 


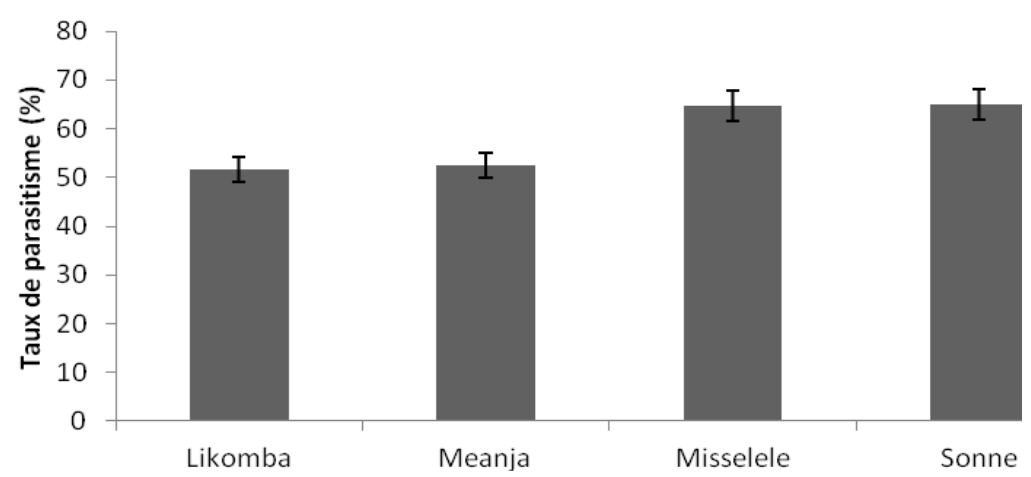

Figure 2. Niveau d'infestation des différents villages

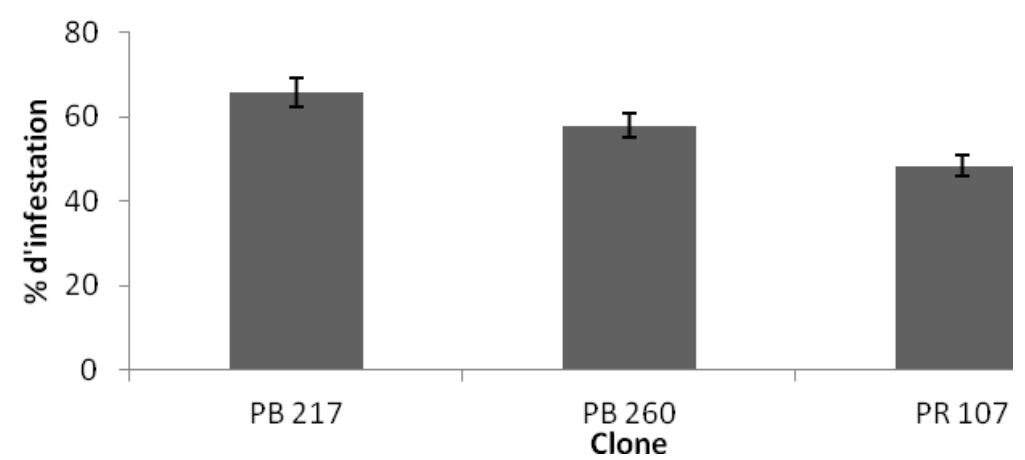

Figure 3. Taux d'infestation des différents clones étudiés

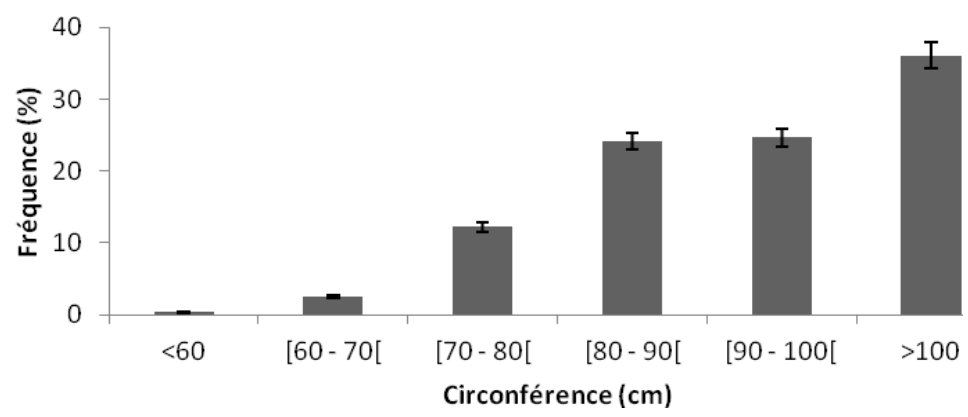

Figure 4. Taux d'infestation de la plantation en fonction de la circonférence des arbres

Intensité du parasitisme : Un total de 7574 touffes a été recensé dans les parcelles étudiées, soit une moyenne de 2,9 touffes par pied d'hévéa. La localité de Meanja est celle qui compte le nombre le plus élevé de touffes par individu $(3,95)$, moyenne significativement élevée à celle rencontrée dans les parcelles de Likomba et Misselele ( $P$ value $<0,001$ ) (Figure 5). Au niveau des clones, le clone PR 107 compte une moyenne de touffes du parasite par individu de 4,38 significativement élevée par rapport au clone PB 260, qui a une moyenne de 2,61 touffes du parasite par arbre (Figure 6). L'analyse des droites de régression du nombre de touffes du parasite en fonction de la circonférence de l'arbre dans les différentes parcelles montre qu'il existe un lien entre ces deux paramètres (Figure 7). En effet, le nombre de touffes du parasite augmente avec la circonférence de l'arbre. 


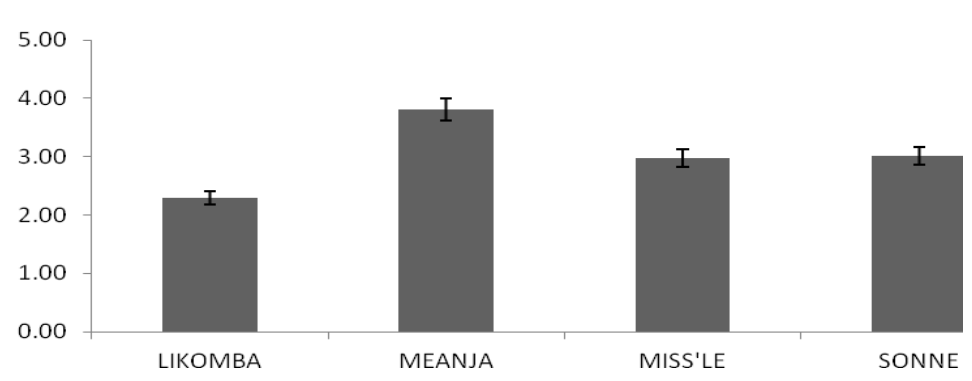

Figure 5. Nombre de touffes du parasite par arbre dans les villages prospectés

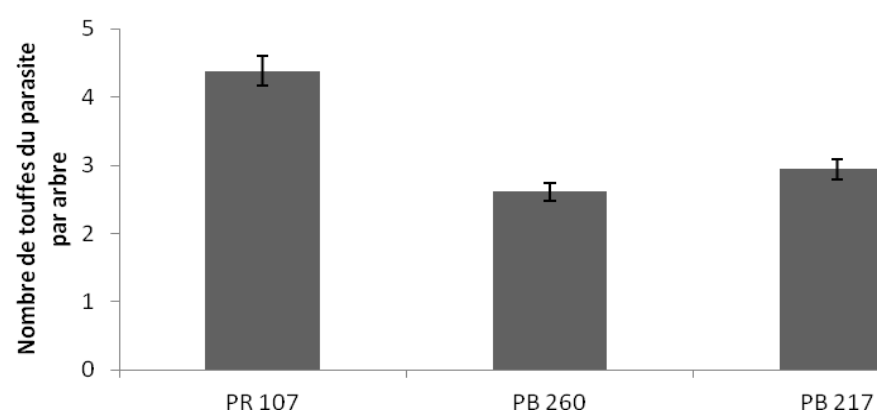

Figure 6. Nombre de touffes du parasite par arbre chez les clones étudiés
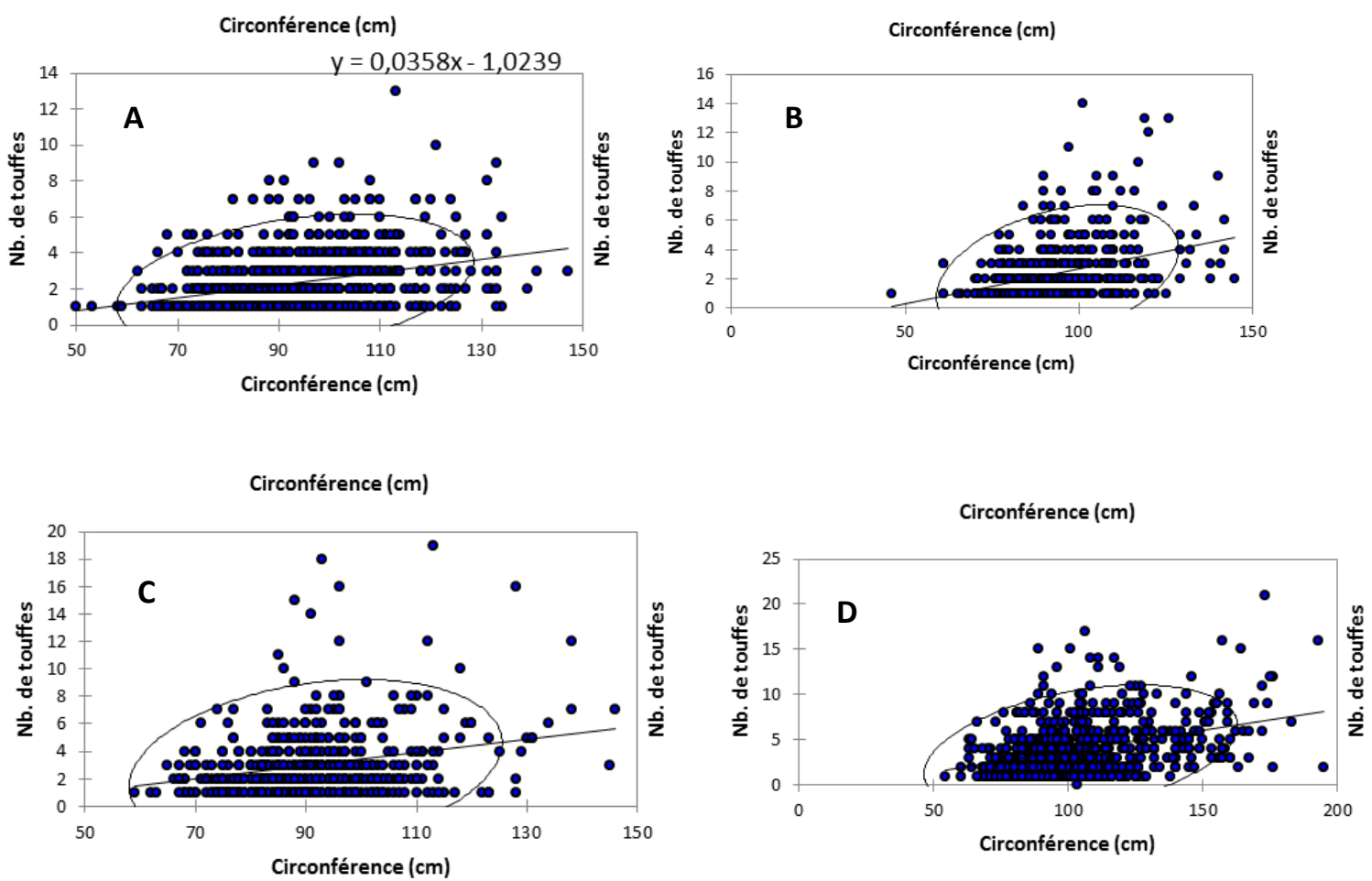

Figure 7 : Droites de régression du nombre de touffes de Loranthaceae en fonction de la circonférence de l'arbre dans les villages prospectés. A : Likomba ; B : Meanja ; C : Misselele ; D : Sonne 


\section{Ngotta Biyon Bi et al. J. Appl. Biosci. 2015 Niveau de parasitisme des hévéas par les Loranthaceae dans la région du Sud-ouest Cameroun}

Distribution du parasitisme : Le taux de parasitisme diminue de la bordure vers l'intérieur de la plantation (Figures 8 et 9). La parcelle de Sonne est celle dont la bordure est la plus parasitée, avec un taux de 61,23\%, tandis que celles de Likomba et Misselele possèdent les bordures les moins parasitées $(43,18 \%)$. II a été également constaté que les routes principales présentent un taux de parasitisme significativement plus élevé que les routes secondaires et les routes tertiaires, et ces différences sont significatives $(P$ value $=0,012)$. De même, les individus situés en bordure présentent la plus grande intensité de parasitisme. En effet, dans toutes les parcelles étudiées, le nombre de touffes du parasite par individu est le plus élevé en bordure (Figure 10). La bordure de la parcelle de Sonne possède le nombre moyen de touffes du parasite le plus élevé, avec une moyenne de 4,34. Par contre la bordure de la parcelle de Likomba possède le nombre de touffes par individu le moins élevé $(2,49)$.

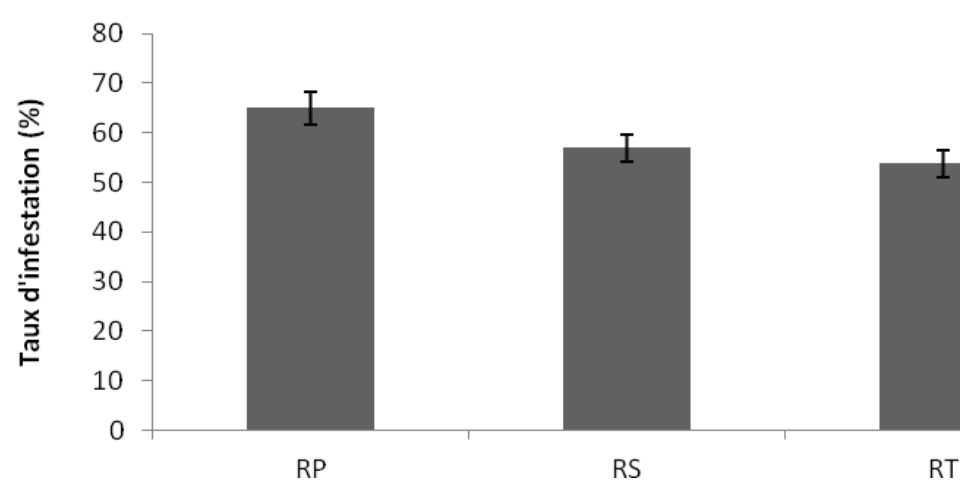

Figure 7: Taux d'infestation des différents axes étudiés. RP: Route principale; RS: Route secondaire; RT: Route tertiaire.

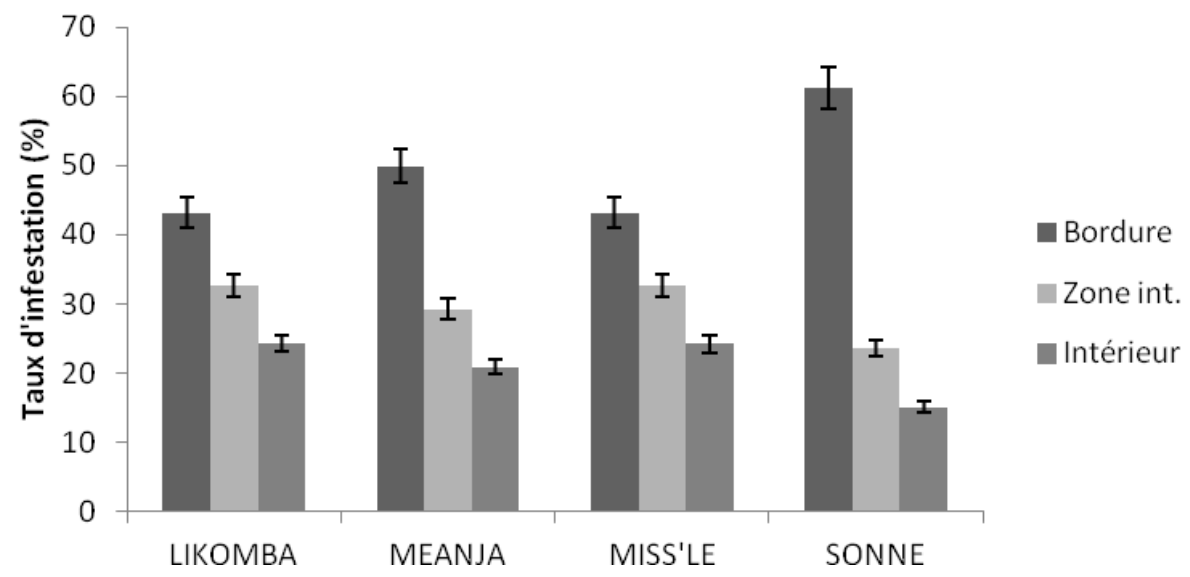

Figure 8 : Taux d'infestation des différentes parcelles. 


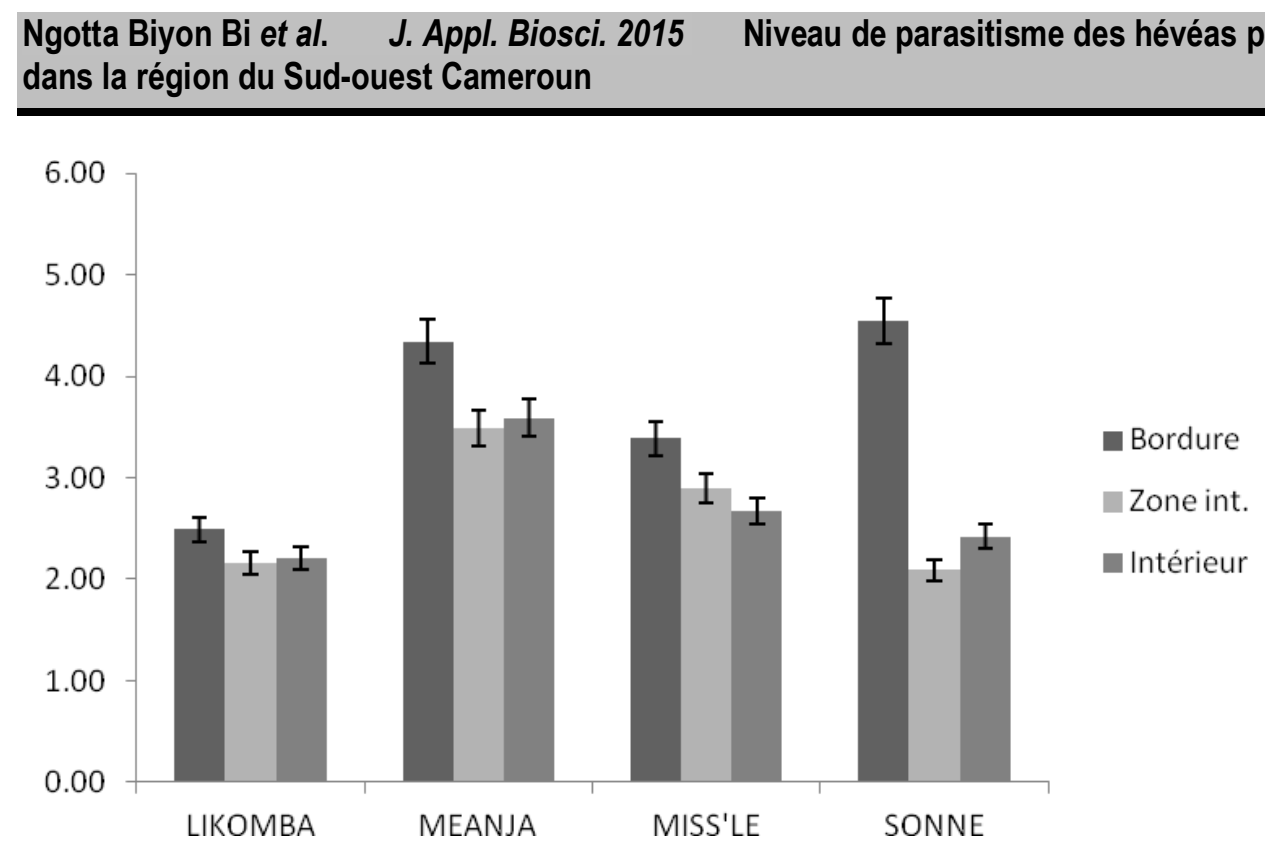

Figure 9 : Nombre de touffes du parasite par arbre dans les villages étudiés

\section{DISCUSSION}

Loranthaceae rencontrée: Une seule espèce de Loranthaceae a été rencontrée dans les parcelles de la CDC, Phragmanthera capitata. Cette plante parasite, abondante dans la région du sud-ouest est signalée dans les plantations d'hévéas dans le sud du Cameroun, au Gabon, en Côte-d'Ivoire et au Benin (Engone Obiang \& Sallé, 2006 ; Dibong et al., 2009 ; Soro et al., 2010 ; Houenon et al., 2012). Ondoua et al. (2015) ont trouvé un taux de parasitisme maximum de $17,48 \%$ chez les cacaoyers dans le Sud-Cameroun. Cette différence par rapport au taux de parasitisme observé dans le sud-ouest s'explique par le fait que le parasitisme varie en fonction de la position géographique et des paramètres floristiques de l'hôte (Dibong et al., 2009; Raynald Roques \& Paré, 1996 cit. Fotso et al., 2014).

Niveau de parasitisme des plantations: L'étude a révélé un taux de parasitisme de $51,36 \%$. Soro et al. (2010) ont trouvé un taux de 32,40\% chez les hévéas âgés de 15 à 20 ans. Ceci peut s'expliquer par le fait que le parasitisme augmente avec l'âge de l'individu (Engone obiang, 2002). Le taux de parasitisme varie significativement du village Sonne au village Misselele. Cette variation peut s'expliquer par le fait que les parcelles du village Sonne sont situées le long de la route principale, tandis que celles du village Misselele sont situées le long des routes secondaires et tertiaires. Ces différents axes, de largeur variable, ont donc influencé le parasitisme. Cette observation permet de confirmer l'implication anthropique dans la dissémination des Loranthaceae (Dibong et al., 2009). Les résultats ont montré que les individus possédant les plus grandes circonférences sont les plus parasités. Ces résultats sont en accord avec ceux trouvés au Gabon (Engone Obiang \& Sallé, 2006). Ces auteurs pensent que les arbres à plus gros diamètre étant certainement les plus élevés, constitueraient un support de choix pour les disséminateurs aviaires des baies des Loranthaceae. Le clone PB 260 présente un taux de parasitisme de 57,84 $\%$, ce qui est largement supérieur à la valeur trouvée au Gabon qui était de $19 \%$ (Engone Obiang, 2002). Cette différence peut s'expliquer par le fait que le parasitisme dépend de la position géographique et des paramètres floristiques de l'hôte (Dibong et al., 2009). Ainsi, le fait qu'un individu soit situé sur la côte, ou qu'il soit situé à l'intérieur du continent peut affecter le parasitisme.

Distribution du parasitisme : L'étude sur la distribution du parasitisme a révélé que les individus situés en bordure de route sont les plus parasités. Cette observation a également été faite par Soro et al. (2010) sur les plantations d'hévéas en Côte-d'Ivoire. Ceci s'expliquerait par le fait que le milieu soit plus ouvert en bordure de route qu'à l'intérieur de la plantation. Les bordures de plantations constitueraient donc un endroit propice pour l'installation de $P$. capitata qui est une espèce héliophile (Dibong et al., 2009). Traoré et al. (2003) trouvent que les plantes de bordure sont proches des foyers de dissémination des oiseaux frugivores, donc favorables au parasitisme. Cette étude a également montré des différences significatives du taux de parasitisme entre les routes principales et les routes secondaires et tertiaires. La largeur des voies d'accès influence le déplacement des disséminateurs des 


\section{Ngotta Biyon Bi et al. J. Appl. Biosci. 2015 Niveau de parasitisme des hévéas par les Loranthaceae dans la région du Sud-ouest Cameroun}

Loranthaceae. Ainsi, plus une voie d'accès est grande, plus le parasitisme est élevé. Une des solutions pour

\section{CONCLUSION}

L'étude menée dans les plantations d'hévéas de la région du sud-ouest a révélé que les Loranthaceae constituent une menace réelle pour celles-ci. Une seule espèce a été recensée à savoir Phragmanthera capitata, qui est signalée comme majoritaire dans toute la partie sud du Cameroun. Le taux de parasitisme de la plantation est globalement élevé, et le clone PB 217 est le plus

\section{REMERCIEMENTS}

Les remerciements vont à l'encontre de la Cameroon Development Coorporation $(C D C)$ qui ont mis à notre

\section{REFERENCES}

Bannister P, Graham L, Strong, Inge A, 2002. Differential accumulation of nutrient elements in some new mistletoes and their hosts, Functional Plant Biology 29, 11: 1309-1318.

Boussim J, 2002. Les phanérogames parasites du Burkina Faso: inventaire, taxonomie, écologie et quelques aspects de leur biologie. Cas particulier des Loranthaceae parasites du karité. Thèse de doctorat d'état, Université de Ouagadougou, $285 \mathrm{p}$.

Dibong SD, Engone Obiang NL, Din N, Priso RJ, Taffouo VD, Fankem H, Amougou A, 2009. Niveau d'infestation des arbres fruitiers des groupements végétaux par Phragmanthera capitata (Sprengel) S. Balle (Loranthaceae) dans la région littorale du Cameroun. Int. J. Biol. Chem. Sci. 3, 2:347-454.

Engone Obiang NL and Sallé G, 2006. Faut-il éradiquer Phragmanthera capitata, parasite des hévéas en Afrique ? C. R. Biologies 329 : 185-195.

Fotso, Mbouobda HD, Tita MA, Muyang RF, Belfiang ND, Omokolo ND, 2014. Parasitism of plum tree (Dacryodes edulis) by Loranthaceae in FotetsaDschang (West Cameroon). African Journal of Agricultural Research 9, 29 : $2255-2262$.

HEVECAM (Hévéa du Cameroun), 1995. Rapport de la Direction Exploitation Agricole : Bilan de l'essai Loranthus. 12 AGRO/DEA. 9 p.

Houenon JG, Yedomonhan H, Adomo AC, Tossou MG, Omorou M, Akoegninou A, 2012. Les Loranthaceae des zones guinéenne et soudanoguinéenne au Bénin et leurs hôtes. Int. J. Bio. Chem. Sci. 6, 4 : 1669 - 1686. réduire le parasitisme dans ces plantations serait de planter les clones résistants en bordure de route.

parasité. II a également été montré que les individus situés en bordure de plantation sont plus parasités que ceux situés à l'intérieur. D'autre part, les routes principales ont montré un taux de parasitisme plus élevé que les routes secondaires et tertiaires. Le clone PR 107 qui est le moins parasité, pourrait être utilisé en bordure de route, afin de limiter la propagation du parasitisme.

disposition leurs plantations ainsi que leur appui logistique pour le bon déroulement de ce travail.

NL Engone Obiang, 2002. Les Loranthaceae au Gabon. Mémoire de diplôme d'études supérieures, Université Pierre et Marie Curie, Paris. $61 \mathrm{p}$.

Ondoua JM, Dibong SD, Taffouo VD, Ngotta Biyon JB, 2015. Parasitisme des champs semenciers de cacaoyers par les Loranthaceae de la localité de Nkoemvone (Sud Cameroun). Journ. of Appl. Biosc. 85 : 1774 - 1803.

Polhill R, and Wiens D, 1998. Mistletoes of Africa. The Royal Botanic Kew, $370 \mathrm{p}$.

Raynal-Roques A and Paré J, 1998. Biodiversité des Phanérogames parasites: leur place dans la classification systématique. Adansonia 20: 313322.

Sonké B, Kenfack D, Tindo M, 2000. Parasitisme de l'avocatier (Persea americana, Lauraceae) par les Loranthacées dans la région de Yaoundé (Cameroun). Fruits 55 : 325-331.

Soro K, Soro D, N'Guessan K, Gnahoua GM, Traoré D, 2010. Parasitisme des Loranthaceae sur les hévéas en zone forestière des sous-préfectures de Gagnoa et d'Ouragahio, en Côte d'Ivoire. Journal of Animal and Plant Sciences, 6 : 597604. 